\title{
Never-smoker African-American women suffer more from COPD compared with never-smoker Caucasian women
}

To the Editor:

The article by GuT-Gobert et al. [1] presents an excellent review of evolving challenges in the management and prevention of COPD in women.

The population of African-American women in western Europe is substantial and growing. We wish to point out a few puzzling and paradoxical findings about the epidemiology of COPD in this subpopulation in the USA that may have relevance for the European situation.

Gut-Gobert et al. [1] suggest that, between 1990 and 2010, COPD became the third leading cause of death in the USA. The COPD mortality rate declined among men in the USA between 1999 (57.0 per 100 000) and 2006 (46.4 per 100000), whereas there was no significant change in mortality rates for women ( 35.3 per 100000 in 1999 and 34.2 per 100000 in 2006).

It is notable that the COPD mortality rates are lower in African-American than Caucasian women; however, asthma prevalence and mortality rates are higher in African-American women than Caucasian women [2].

Paradoxically, several population-based surveys found a higher prevalence of self-reported COPD diagnosis in non-Hispanic African-American than in non-Hispanic Caucasian women. For example, in the nationwide Behavioral Risk Factor Surveillance System (BRFSS) study, COPD was defined by an affirmative response to the question: "Has a doctor, nurse or other health professional ever told you that you have chronic obstructive pulmonary disease or COPD, emphysema, or chronic bronchitis?" Cross-sectional data from 405285 US adults aged $\geqslant 18$ years in the 2011 BRFSS are shown in figure 1 [3]. Overall, 17.5\% reported being current cigarette smokers, 6.9\% reported having COPD and 71.2\% reported another chronic condition [4]. The prevalence of COPD diagnosis in never-smokers was higher in African-American than in Caucasian women.

What factors could explain a higher prevalence of COPD among African-American than Caucasians never-smokers? The higher prevalence of reported diagnoses of COPD in African-American never-smoker women compared with Caucasian never-smoker women (figure 1) might be explained by increased exposure to second-hand smoke (SHS) in African-American women [5-7]. Low socioeconomic conditions may also lead to heightened exposure of environmental toxins, such as indoor and outdoor air pollution. Other contributing factors are occupational exposure, prior exposure to biomass fuel among immigrants $[5,6,8]$ along with chronic asthma [9], which is much higher in African-Americans. In general, women are reported to be $50 \%$ more likely to have COPD, even when adjusted for height, education, income and healthcare access [5]. Sex hormones may also have a role [5]. Metabolism of cigarette smoke varies according to race; nicotine has a higher half-life in African-Americans compared with Caucasians [7].

Contrary to reported diagnoses, COPD mortality rates are lower in African-American than Caucasian women [10]; however, asthma prevalence and mortality rates are higher in African Americans than Caucasians. Evolution of asthma to COPD, underdiagnosed chronic airway disease, or the mixed syndrome of asthma and COPD could relate to an apparent higher prevalence in African-American women [2]. A recent analysis of SHS exposure using serum cotinine showed a greater prevalence of

@ERSpublications

The prevalence of COPD is increasing; it will be the third leading cause of mortality in the USA by this year, 2020. There is a puzzling increasing trend of COPD in never-smoker black women, which may have relevance for the European situation. http://bit.ly/2ZLkOzR

Cite this article as: Sumon M, Mehari A. Never-smoker African-American women suffer more from COPD compared with never-smoker Caucasian women. Eur Respir Rev 2020; 29: 190173 [https://doi.org/ 10.1183/16000617.0173-2019]. 
FIGURE 1 COPD according to race and sex in never-smokers. Behavioral Risk Factor Surveillance System 2011. Data from [3].

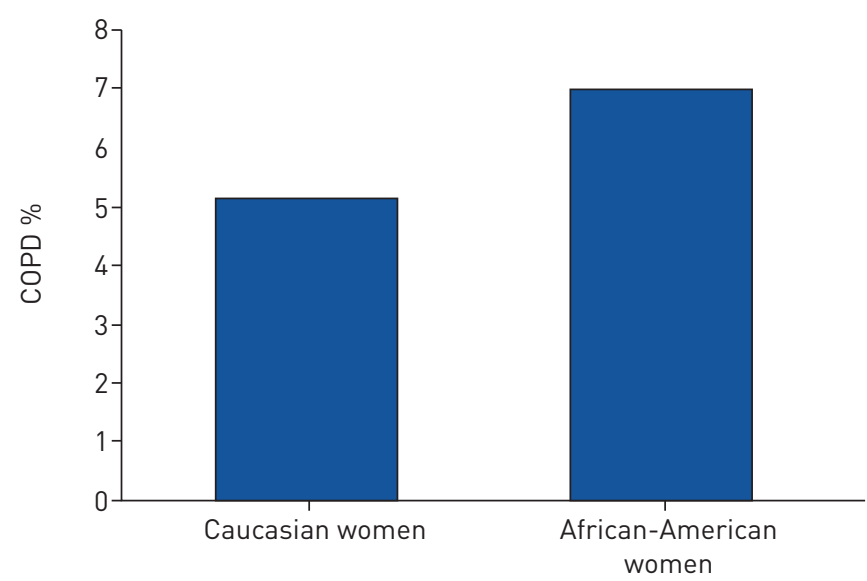

exposure in non-Hispanic African-Americans than non-Hispanic Caucasians or Mexican-Americans [7]. Further, non-Hispanic African-American children aged 3-11 years had the highest exposure prevalence; thus, SHS exposure early in life may also contribute to the finding in African-American women.

Multivariate regression analysis of National Health and Nutrition Examination Survey (NHANES) III sampled populations did not show any racial differences in the rate of airway obstruction; rather, this was mostly influenced by increasing age, male sex and a lower body mass index [4].

Further research for identification of the causes of COPD in never-smokers would help create a more effective COPD prevention strategy in never-smokers.

Mahbubur Sumon and Alem Mehari

Division of Pulmonary Critical Care Medicine, Howard University Hospital, Washington, DC, USA.

Correspondence: Mahbubur Sumon, Division of Pulmonary Critical Care Medicine, Howard University Hospital, 2041 Georgia Ave, Suite 4F03, Washington, DC 20060-0002, USA. E-mail: msumon@huhosp.org

Received: 13 Dec 2019 | Accepted: 18 Dec 2019

Provenance: Submitted article, peer reviewed

Conflict of interest: None declared.

\section{References}

1 Gut-Gobert C, Cavaillès A, Dixmier A, et al. Women and COPD: do we need more evidence? Eur Respir Rev 2019; 28: 180055.

2 Grant EN, Lyttle CS, Weiss KB. The relation of socioeconomic factors and racial/ethnic differences in US asthma mortality. Am J Public Health 2000; 90: 1923-1925.

$3 \mathrm{Xu} \mathrm{F}$, Mawokomatanda T, Flegel D, et al. Surveillance for certain health behaviors among states and selected local areas - Behavioral Risk Factor Surveillance System, United States, 2011. MMWR Surveill Summ 2014; 63: 1-149.

4 Celli BR, Halbert RJ, Nordyke RJ, et al. Airway obstruction in neversmokers: results from the Third National Health and Nutrition Examination Survey. Am J Med 2005; 118: 1364-1372.

5 Fuller-Thomson E, Chisholm RS, Brennenstuhl S. COPD in a population-based sample of never-smokers: interactions among sex, gender, and race. Int J Chronic Dis 2016; 2016 : 5862026.

6 Hagstad S, Ekerljung L, Lindberg A, et al. COPD among non-smokers - report from the obstructive lung disease in Northern Sweden (OLIN) studies. Respir Med 2012; 106: 980-988.

7 Pérez-Stable EJ, Herrera B, Jacob P 3rd, et al. Nicotine metabolism and intake in black and white smokers. JAMA 1998; 280: 152-156.

8 Salvi SS, Barnes PJ. Chronic obstructive pulmonary disease in non-smokers. Lancet 2009; 374: $733-743$.

9 Wheaton AG, Liu Y, Croft JB, et al. Chronic obstructive pulmonary disease and smoking status - United States, 2017. MMWR Morb Mortal Wkly Rep 2019; 68: 533-538.

10 Kamil F, Pinzon I, Foreman MG. Sex and race factors in early-onset COPD. Curr Opin Pulm Med 2013; 19: 140-144. 